PSICOLOGÍA

IBEROAMERICANA
Psicología Iberoamericana

ISSN: 1405-0943

revista.psicologia@ibero.mx

Universidad Iberoamericana, Ciudad de México México

\title{
Propiedades psicométricas de una Escala de Compasión Hacia los Animales (ECA)
}

López Tello, Alejandro; Mondragón Gómez, Raquel; Moreno Coutiño, Ana Beatriz

Propiedades psicométricas de una Escala de Compasión Hacia los Animales (ECA)

Psicología Iberoamericana, vol. 29, núm. 2, e292412, 2021

Universidad lberoamericana, Ciudad de México, México

Disponible en: https://www.redalyc.org/articulo.oa?id=133967822006

DOI: https://doi.org/10.48102/pi.v29i2.412

Los autores/as conservan los derechos de autor y ceden a la revista el derecho de la primera publicación, con la obra registrada bajo la Licencia Creative Commons Atribución 4.0 Internacional.

Los autores/as conservan los derechos de autor y ceden a la revista el derecho de la primera publicación, con la obra registrada bajo la Licencia Creative Commons Atribución 4.0 Internacional.

\section{(c) (1)}

Esta obra está bajo una Licencia Creative Commons Atribución 4.0 Internacional. 
Artículos

Propiedades psicométricas de una Escala
de Compasión Hacia los Animales (ECA)

Psychometric properties of the Scale of Compassion Towards Animals (ECA)

Alejandro López Tello alejandrolopez2024@gmail.com Universidad Iberoamericana, Ciudad De México , México

(iD) https://orcid.org/0000-0002-1636-6504

Raquel Mondragón Gómez raquemg89@gmail.com

Universidad Nacional Autónoma de México (UNAM), México

(D) https://orcid.org/0000-0002-1785-3291

Ana Beatriz Moreno Coutiño moca99_99@yahoo.com Universidad Nacional Autónoma de México (UNAM), México

(D) https://orcid.org/0000-0002-1601-9318

Psicología Iberoamericana, vol. 29, núm. 2, e292412, 2021

Universidad Iberoamericana, Ciudad de México, México

Recepción: 09 Agosto 2021

Aprobación: 21 Octubre 2021

DOI: https://doi.org/10.48102/ pi.v29i2.412

Redalyc: https://www.redalyc.org/ articulo.oa?id=133967822006
Resumen: Humanos y animales se influyen mutuamente en una relación dinámica que deriva en situaciones benéficas o perjudiciales para ambos, por ello el estudio de las variables involucradas en esta relación adquiere relevancia. El presente estudio tuvo por objetivo evaluar las propiedades psicométricas de una Escala de Compasión hacía los Animales (ECA) en población mexicana. La investigación tuvo un diseño ex post facto, por medio de un muestreo no probabilístico se reclutaron 386 participantes hombres y mujeres, mayores de 18 años, a quienes se les aplicó la ECA. El análisis de datos mostró una adecuada consistencia interna $(\alpha=.93)$ y una estructura unifactorial que explicó el 63\% de la varianza. El análisis factorial confirmatorio arrojó adecuados índices de bondad de ajuste $(\chi 2$ entre los grados de libertad $=64.158 / 24=2,67$, CFI $=, 95$, $\mathrm{RMSEA}=, 07, \mathrm{NFI}=.96 ; \mathrm{IFI}=.97)$. En conclusión la ECA es un instrumento adecuado para evaluar compasión hacia los animales en población mexicana.

Palabras clave: compasión, empatía, animales, propiedades psicométricas, confiabilidad. Abstract: Humans and animals influence each other in a dynamic relationship that leads to beneficial or detrimental situations for both. Therefore, the study of the variables involved in this relationship is relevant. The aim of the present study was to evaluate the psychometric properties of an Animal Compassion Scale (ECA) in a Mexican population, comprising of different states. The research had an ex post facto design, by means of a non-probabilistic sampling. Participants included 386 men and women over the age of 18 years and these participants were administered the ECA. The statistical data analysis showed adequate internal consistency $(\alpha=.93)$, a unifactorial structure that explained $63 \%$ of the variance and the confirmatory factor analysis yielded adequate goodness-of-fit indices $(\chi 2$ between degrees of freedom $=64,158 / 24=2.67$, CFI $=, 95$, $\mathrm{RMSEA}=, 07, \mathrm{NFI}=.96$; IFI $=.97)$. In conclusion, the RCT is an adequate instrument to evaluate compassion towards animals in the Mexican population.

Keywords: compassion, empathy, animals, psychometric properties, reliability. 


\section{Introducción}

La compasión es un concepto sobre el que es posible identificar diferentes conceptualizaciones y análisis que derivan de diversas vertientes filosóficas y de pensamiento (Goetz et al., 2010). Actualmente la psicología basada en evidencias ha retomado el concepto para su estudio sistemático y ha definido el constructo como la conciencia del sufrimiento de otros y el subsecuente deseo de aliviarlo e involucra sentimientos de bondad y comprensión sin prejuicios (Bonermann \& Singer, 2013; Goetz et al., 2010; López-Tello \& Moreno-Coutiño, 2019; Ozawa-de Silva et al., 2012; Singer \& Kilmecki, 2014). Los especialistas sobre el tema coinciden en señalar que la compasión forma parte de un conjunto de comportamientos que ayudan a la sobrevivencia de los miembros de una especie y se relaciona con la capacidad de establecer vínculos mediados por respuestas afectivas en mamíferos (Gilbert, 2013; López-Tello \& Moreno-Coutiño, 2019; Ozawa-de Silva et al., 2012; Singer \& Kilmecki, 2014).

Esta conceptualización de compasión tiene su origen en el intercambio filosófico que se ha producido en los últimos años entre la ciencia occidental y la filosofía budista (Neff et al., 2003). Se aleja de definiciones que relacionan el constructo con sentimientos de lástima o pena hacía el otro, las cuales implican colocarse en una posición superior con respecto al otro que sufre. También difiere de las definiciones más cercanas a la etimología de la palabra compasión en occidente la cual enfatiza la posibilidad únicamente de sufrir con o compartir el sufrimiento y no considera la generación de la motivación para aliviarlo como parte del concepto.

La compasión entendida de este modo se ha incorporado gradualmente a la psicología occidental (Germer \& Siegel, 2012) y aunque en un principio se discutía su semejanza con conceptos como bondad o empatía (Phillips \& Taylor, 2009) poco a poco los avances realizados por los principales investigadores sobre el tema en diversos países como Estados Unidos (Davidson \& Harrington, 2002; Neff et al., 2003; Ozawa-de Silva et al., 2012), Reino Unido (Gilbert, 2013) y Alemania (Singer \& Kilmecki, 2014) han mostrado que la compasión es un fenómeno psicológico diferenciado claramente de otros procesos. En América Latina, la investigación sobre el tema aún es incipiente y actualmente los antecedentes de estudios empíricos relacionados de manera más directa con esta conceptualización y propuesta de estudio se encuentran en los trabajos realizados en México por López-Tello y Moreno-Coutiño (2019) y Mercadillo (2012).

Una de las confusiones más frecuentes sobre la compasión se da con el concepto de empatía, esto es entendible, ya que son fenómenos psicológicos cercanos que comparten la reacción afectiva ante el sufrimiento de otro ser, sin embargo, no debe perderse de vista que se trata de procesos esencialmente diferentes y dichos conceptos no deben usarse indistintamente (Germer \& Siegel, 2012; Mercadillo, 2012). La empatía hacía el sufrimiento se refiere únicamente a la 
capacidad de resonar afectivamente con el dolor del otro, esta no implica la motivación de ayudar a aliviar el sufrimiento del otro, en contraparte la compasión conlleva necesariamente la motivación de aliviar el sufrimiento e involucra la activación de redes neurales relacionadas con estados afectivos que no se activan cuándo únicamente se produce una reacción empática (Gilbert \& Choden, 2013; Singer \& Kilmecki, 2014). En este sentido la empatía hacia el sufrimiento se puede considerar un componente afectivo presente en el fenómeno de la compasión (Mercadillo, 2012).

Hasta ahora la investigación sobre compasión se ha centrado en el estudio del fenómeno entre seres humanos, sin embargo, autores como Ricard (2017), señalan la relevancia de profundizar en el estudio de la compasión hacía los animales, la cual parte del desarrollo de una conciencia de igualdad entre humanos y animales (por su condición como seres sintientes), involucra el reconocimiento de cualidades psicológicas en especies animales no humanas y se manifiesta como la motivación de evitar o aliviar el sufrimiento animal.

Esta conceptualización de compasión hacia los animales es relativamente reciente y existen pocas investigaciones que la retomen, sin embargo, es posible encontrar estudios cercanos a este concepto los cuales señalan, que cuando las personas consideran que existen similitudes entre ellas y los animales, muestran mayores niveles de empatía hacia estos y es más probable que manifiesten conductas orientadas a su protección y cuidado (Allen et al., 2002; Butterfield et al., 2012; Kiesler et al., 2006).

Otras investigaciones han explorado la relación que existe entre la empatía dirigida hacia humanos y animales, encontrando por ejemplo, que una mayor empatía y preocupación por el bienestar animal se correlaciona con una mayor presencia de estos comportamientos hacia otros seres humanos (Preylo \& Arikawa, 2008; Signal \& Taylor, 2007; Taylor \& Signal, 2005). Estos datos con consistentes con investigaciones que han mostrado que la presencia de conductas de violencia, maltrato y crueldad animal pueden ser predictoras de estas mismas conductas hacía seres humanos (Ascione \& Shapiro, 2009; Henderson et al., 2011; McPhedran, 2009; Overton et al., 2012; Schwartz et al., 2012).

Aunque estos reportes han comenzado a explorar la dinámica de la relación entre el ser humano y los animales a partir de variables como la empatía, la percepción de cercanía o el trato hacía los animales (Amiot \& Bastian, 2015; Andrukonis et al., 2020; Thompson \& Gullone, 2003), hasta ahora son escasos los estudios empíricos específicos sobre compasión hacía los animales desde la perspectiva de la cual partimos.

Entre las referencias más cercanas al presente trabajo, se encuentran los reportes de Hawkins y Williams $(2016,2017)$, quienes utilizan una escala de compasión hacía los animales en niños, e indican que esta variable se relaciona positivamente con las actitudes e interacciones positivas hacía los animales, aunque es importante señalar que en estos dos trabajos, la compasión se aborda como un objetivo secundario y los autores proporcionan poca información sobre el instrumento utilizado y sus propiedades psicométricas. 
Actualmente es posible encontrar diferentes escalas que miden la compasión hacia los seres humanos (Chang et al., 2014; López-Tello \& Moreno-Coutiño, 2019; Martins et al., 2013; Sprecher \& Fehr, 2005), de las cuales únicamente la Escala de Compasión para población Mexicana ([ECOM], López-Tello \& Moreno-Coutiño, 2019) incorpora un factor que evalúa compasión hacía los animales. Consideramos que el estado de la investigación sobre el tema, presenta una gran área de oportunidad, ya que investigar esto permitiría mejorar la comprensión de las relaciones humano-animal y humano-humano (Amiot \& Bastian, 2017; Bastian, Costello et al., 2012; Caviola et al., 2019; Costello \& Hodson, 2010; Dhont et al., 2014; Fine, 2010; Herzog, 2011).

Estudiar el papel de la compasión hacia los animales también permitiría profundizar en la identificación de los beneficios derivados de una relación positiva con los animales, en esta área de investigación sobresalen los reportes que señalan: 1) beneficios a la salud física y mental en humanos que establecen relaciones significativas con sus mascotas, (Odendaal \& Lehmann, 2000; Marinelli et al., 2007; Schöberl et al., 2012) y 2) menores indicadores de maltrato y sufrimiento animal (Kielland et al., 2010).

Aunado a este último punto, es pertinente considerar que en la actualidad el estudio de las relaciones que establece el ser humano con el medio ambiente es cada vez más importante debido a la relevancia del factor humano en el surgimiento de una serie de problemas que amenazan el equilibrio de los ecosistemas y la vida de diversas especies en el planeta (Gamboa-Bernal, 2011; Valera, 2019). En este sentido, el estudio de la compasión hacia los animales puede ser clave no solo para mejorar la relación entre el ser humano y otras especies animales, sino que puede representar un elemento central en el desarrollo de estrategias y políticas de conservación ambiental (Bekoff, 2010, 2013; Ramp \& Bekoff, 2015).

Debido a la relevancia del constructo la presente investigación tuvo por objetivo el análisis de las propiedades psicométricas de una escala de compasión hacía los animales en población mexicana. Esta investigación es una ampliación de la primera aproximación realizada en la ECOM (López-Tello \& Moreno-Coutiño, 2019) y tiene por objetivo evaluar las características psicométricas de un instrumento específico que evalué compasión hacia los animales en población mexicana, con base en esto, se identifican las siguientes hipótesis como aquellas que permitieron el cumplimiento del objetivo:

Hipótesis 1. La Escala de Compasión hacía los Animales (ECA) tendrá una estructura factorial unidimensional con una carga factorial $\geq .50$ en cada ítem, y sus principales índices de adaptación serán suficientes (raíz cuadrada media error de aproximación $[\mathrm{RMSEA}]<.08$, índice de ajuste comparativo $[\mathrm{CFI}]>.80)$.

Hipótesis 2. La ECA tendrá una consistencia interna media-alta (alfa de Cronbach $>.70$ ), esto considerando los antecedentes del trabajo realizado por López-Tello y Moreno-Coutiño (2019).

Hipótesis 3. La ECA se correlacionará positivamente con los puntajes de la ECOM (López-Tello \& Moreno-Coutiño, 2019). Esta hipótesis 
derivada de los datos que ya reportados en la ECOM, trabajo del cual se desprende este estudio.

\section{Método}

\section{Tipo de estudio}

El estudio es no experimental, exploratorio, transversal, con un diseño ex post facto, que es una metodología empírico-analítica en la que el investigador no tiene la intención de manipular variables ni modificar el fenómeno objeto de estudio, sino que se limita a medirlo (Vega, 2015).

\section{Participantes}

Para el presente estudio se utilizó un método no probabilístico de autoselección por individuos, es decir, los participantes no fueron previamente seleccionados sino que al recibir una invitación ellos decidían participar o no; este proceso se llevó a cabo a través de medios electrónicos (celular y computadora). Los participantes recibían un mensaje a su correo electrónico o por medio de WhatsApp en el que se les explicaba de manera breve el objetivo de la investigación y la posibilidad de participar. Este mensaje incluía un enlace el cual redirigía al participante a un formulario electrónico elaborado en la plataforma google forms y en el que se describía el objetivo de la investigación, el contenido de los cuestionarios que debían responder y la solicitud de acuerdo expreso para participar de manera voluntaria garantizando en todo momento el anonimato y la confidencialidad de los datos. Si el participante aceptaba participar se desplegaba el cuestionario, brindándole la posibilidad de detenerse en cualquier momento que deseara, al llegar al final del cuestionario, se abría una pantalla de agradecimiento por la participación en el estudio, es pertinente mencionar que no se realizó ningún pago a los participantes, condición que era especificada antes de que las personas decidieran participar en el estudio.

Para el análisis factorial exploratorio se reclutaron 90 participantes de nacionalidad mexicana, posteriormente para la segunda fase del estudio en que se realizó un análisis factorial confirmatorio se reclutaron 296 participantes. Como criterios de inclusión los participantes seleccionados debían tener al menos 18 años de edad, ser de nacionalidad mexicana y estar de acuerdo en participar en el estudio.

En la primera muestra para el análisis factorial exploratorio, los participantes tuvieron una edad promedio de 41.49 años, 66 (73.3\%) participantes eran mujeres, 24 (26.7\%) eran hombres, en relación al nivel educativo la mayoría contaban con estudios de nivel licenciatura o superior $(85.5 \%)$ y en relación a la ciudad de origen de estos participantes, la mayoría eran de la Ciudad de México (66.7\%) y del Estado de México (8.9\%), los datos de esta muestra fueron colectados entre los meses de enero y marzo de 2019. En la Tabla 1 se pueden observar con mayor 
detalle las características sociodemográficas de los participantes de esta muestra.

\section{Tabla 1}

\section{Tabla 1}

Datos sociodemográficos de la muestra 1

\begin{tabular}{lcc}
\hline & Frecuencia $(\mathrm{n}=90)$ & Porcentaje \\
\hline Sexo & 24 & 26.7 \\
Masculino & 66 & 73.3 \\
Femenino & & \\
Nivel de estudios & 77 & 85.5 \\
Licenciatura o superior & 11 & $12, .2$ \\
Nivel medio superior & 2 & 2.2 \\
Secundaria & & \\
Ciudad de origen & 60 & 66.7 \\
Ciudad de México & 8 & 8.9 \\
Estado de México & 6 & 6.7 \\
Coahuila & 4 & 4.4 \\
Nuevo León & 3 & 3.3 \\
Oaxaca & 2 & 2.2 \\
Durango & 1 & 1.1 \\
Baja California & 1 & 1.1 \\
Chiapas & 1 & 1.1 \\
Guanajuato & 1 & 1.1 \\
Guerrero & 1 & 1.1 \\
Hidalgo & 1 & 1.1 \\
Puebla & 1 & 1.1 \\
Querétaro & & \\
& &
\end{tabular}

En la segunda muestra con la cual se llevó a cabo el análisis factorial confirmatorio, los participantes tuvieron una edad promedio de 36.5 años, 206 (69.6\%) eran mujeres, 90 (30.4\%) eran hombres, 214 (72.2\%) contaban con estudios profesionales y pertenecían a diferentes estados de la República Mexicana. Los datos de esta muestra se recolectaron en los meses de noviembre de 2019 a marzo de 2020, en la Tabla 2 se pueden consultar con mayor detalle los datos sociodemográficos de estos participantes. 
Tabla 2

Tabla 2

Datos sociodemográficos de la muestra 2

\begin{tabular}{lcc}
\hline & Frecuencia $(\mathrm{n}=296)$ & Porcentaje \\
\hline Sexo & 90 & 30.4 \\
Masculino & 206 & 69.6 \\
Femenino & & \\
Nivel de estudios & 214 & 72.2 \\
$\quad$ Licenciatura o superior & 74 & 24.9 \\
Nivel medio superior & 8 & 2.7 \\
Secundaria & & \\
Ciudad de origen & 174 & 58.8 \\
Ciudad de México & 51 & 17.2 \\
Estado de México & 43 & 14.5 \\
Veracruz & 7 & 2.4 \\
Coahuila & 4 & 1.4 \\
Querétaro & 2 & .7 \\
Guanajuato & 2 & .7 \\
Hidalgo & 2 & .7 \\
Michoacán & 2 & .7 \\
Puebla & 1 & .3 \\
Colima & 1 & .3 \\
Durango & 1 & .3 \\
Guerrero & 1 & .3 \\
Jalisco & 1 & .3 \\
Morelos & 1 & .3 \\
Nuevo León & 1 & .3 \\
Oaxaca & 1 & .3 \\
Quintana Roo & 1 & .3 \\
San Luis Potosí & & \\
\hline & &
\end{tabular}

\section{Instrumentos}

Escala de Compasión hacía los Animales (ECA): El cuestionario es una escala tipo Likert conformada por nueve reactivos con cinco opciones de respuesta (nunca, casi nunca, a veces, casi siempre y siempre). Los reactivos se obtuvieron de un análisis secundario de la base de datos estructurada originalmente para el desarrollo de la Escala de Compasión para población mexicana (ECOM) de López-Tello y Moreno-Coutiño (2019) del cual retoma tres reactivos que en la ECOM evalúan compasión hacia los animales.

Escala de Compasión para Población Mexicana (ECOM, LópezTello \& Moreno-Coutiño, 2019): Escala tipo Likert conformada por 17 reactivos con cinco opciones de respuesta (nunca, casi nunca, a veces, casi siempre y siempre). La escala reporto una alpha de .90 con una estructura de tres factores que mostró adecuados índices de bondad de ajuste en el análisis factorial confirmatorio. Para el presente estudio se retomaron únicamente 14 reactivos del cuestionario original correspondientes a dos 
de los factores que lo conforman llamados motivación para aliviar el sufrimiento y empatía hacía el sufrimiento. Se contó con la autorización expresa de los autores de la ECOM para hacer uso del instrumento.

\section{Análisis de datos}

Los análisis se realizaron con el paquete SPSS versión 21 y el programa AMOS 24. Se realizaron análisis descriptivos para explorar las características generales de los participantes. Para analizar la consistencia interna del cuestionario se llevó a cabo una prueba de alpha de Chronbach. Para comprobar si era factible realizar un análisis factorial exploratorio, se utilizó el análisis de Kaiser-Meyer-Olkin y para verificar la adecuación del tamaño de la muestra y para comprobar la redundancia entre las variables reunidas entre los factores del instrumento se realizó un análisis de esfericidad de Bartlett. Se realizó un análisis factorial exploratorio mediante el método de extracción de componentes principales y una rotación varimax solicitando al software la eliminación de las cargas factoriales menores a .40 .

Posteriormente se realizó un análisis factorial confirmatorio basado en el método de ecuaciones estructurales (Byrne, 2001; Kline, 2005), para este análisis se fijaron las varianzas de los términos de error como parámetros libres y se utilizó la estimación de máxima verosimilitud. Los índices de bondad de ajuste utilizados fueron la $X^{2}$ (este cociente se dividió entre los grados de libertad y para considerar un buen ajuste el resultado debe ser < 4), el índice de ajuste comparativo de Bentler (CFI) (cuanto más se acerque el valor a .90, mejor será el ajuste) y el error cuadrático medio de aproximación (RMSEA; un ajuste relativo $\geq .90$ se considera generalmente un buen ajuste); el índice de ajuste normalizado (NFI) y el índice de ajuste incremental (IFI) en estos dos indicadores un ajuste relativo $\geq .90$ se considera en general un buen ajuste.

Posteriormente con la finalidad de encontrar evidencias de validez por medio de la correlación del constructo con otras variables, se realizó un análisis de correlación por medio de una prueba de $\mathrm{r}$ de Pearson entre los puntajes de la ECA y las sub-escalas de la ECOM.

\section{Resultados}

\section{Consistencia interna y validez del cuestionario}

A partir del análisis de consistencia interna realizado con la primera muestra se identificó un coeficiente de alpha de Cronbach de .93. El resultado del análisis Kaise-Meyer-Olkin fue .914 y el resultado de la prueba de esfericidad de Bartlett fue de 583.071 ( $\mathrm{df}=36, \mathrm{p}<.001$ ), estos resultados indicaron que era factible realizar un análisis factorial exploratorio (AFE). 
El AFE indicó que una solución de un solo factor conformado por nueve reactivos explica el $64.36 \%$ de la varianza. En la Tabla 3 es posible observar esta información resumida.

\section{Tabla 3}

\section{Tabla 3}

Consistencia interna y análisis factorial exploratorio de la escala de compasión hacía los animales

\begin{tabular}{lc}
\hline Reactivos & $\begin{array}{c}\text { Carga } \\
\text { estandarizada }\end{array}$ \\
\hline 1. Me entristece el sufrimiento de los animales & .873 \\
2. Cuando veo a un animal enfermo siento deseos de ayudarlo & .860 \\
3. Cuando veo a un animal sufriendo maltrato siento deseos de & .851 \\
protegerlo & \\
4. Cuando veo a un animal abandonado siento muchas ganas de & .839 \\
ayudarlo & \\
5. Siento un gran amor por los animales & .816 \\
6. Procuro cuidar de los animales & .809 \\
7. Quisiera que ningún animal sufriera & .754 \\
8. Si veo a un animal en peligro trato de ayudarlo & .744 \\
9. Sé que los animales sufren igual que los seres humanos & .649 \\
Varianza explicada & \\
Alpha de Chronbach & $64.4 \%$ \\
\hline
\end{tabular}

El análisis factorial confirmatorio, reafirmó la estructura de un solo factor encontrada durante el análisis exploratorio de la escala con índices aceptables de bondad de ajuste (ver Tabla 4).

\section{Tabla 4}

Tabla 4

Resultados del Análisis Factorial Confirmatorio

\begin{tabular}{ccc}
\hline Indicador de ajuste & \multicolumn{2}{c}{ Coeficiente } \\
\hline & $\chi^{2 / g l}$ & 2,67 \\
CFI & .95 \\
& RMSEA & .07 \\
NFI & .96 \\
IFI & .97 \\
\hline
\end{tabular}


Por otra parte, se identificó que la puntuación promedio de la ECA obtenida por los participantes del estudio fue de $36.6(\mathrm{sd}=6.8)$, y no se encontraron diferencias significativas en el puntaje de acuerdo al género, ni al nivel educativo de los participantes. En la Tabla 5 es posible observar las puntuaciones promedio obtenidas en la ECA de acuerdo a estas variables de agrupación.

\section{Tabla 5}

\section{Tabla 5}

Puntuaciones promedio y desviación estándar de la ECA por sexo y nivel de estudios

\begin{tabular}{|c|c|}
\hline ECA & $\overline{\mathrm{x}}(\mathrm{sd})$ \\
\hline \multicolumn{2}{|l|}{ Sexo } \\
\hline Masculino & $37(6.9)$ \\
\hline Femenino & $36.5(6.8)$ \\
\hline \multicolumn{2}{|l|}{ Grado académico } \\
\hline Licenciatura o superior & $36.3(6.8)$ \\
\hline Nivel medio superior & $37.7(6.9)$ \\
\hline Secundaria & $34.7(4.8)$ \\
\hline
\end{tabular}

Finalmente se exploró la correlación entre los puntajes obtenidos en la escala de compasión hacia los animales y las sub-escalas del instrumento ECOM en las que en el factor motivación para aliviar el sufrimiento la puntuación promedio fue de 36.2 (sd 5.6) y en el factor empatía hacia el sufrimiento fue de 16.4 (sd 3). Los resultados mostraron una correlación positiva, moderada y estadísticamente significativa entre el puntaje total de la ECA con las sub escalas de motivación para aliviar el sufrimiento $(\mathrm{r}=.360, \mathrm{p}<.01)$ y la empatía hacia el sufrimiento $(\mathrm{r}=.477, \mathrm{p}<.01)$ del instrumento ECOM.

\section{Discusión}

En general los resultados de confiabilidad y validez del presente estudio mostraron que la ECA posee propiedades psicométricas adecuadas para medir compasión hacia los animales en población mexicana. Esto nos permite aceptar las tres hipótesis de investigación planteadas en el presente estudio, ya que: 1) se confirmó una estructura de un solo factor e índices de ajuste aceptables, 2) se obtuvo un coeficiente de consistencia interna superior a .7 y 3 ) se observó una correlación positiva con las subescalas de la ECOM.

La estructura unifactorial del instrumento difiere de estudios previos en los que se han comprobado estructuras de por lo menos dos factores (Chang et al., 2014; López-Tello \& Moreno-Coutiño, 2019; Martins et al., 2013; Sprecher \& Fehr, 2005), sin embargo, es importante considerar que estos estudios tenían como objetivo principal la evaluación de la compasión entre seres humanos. Esto nos permite cuestionar si la investigación sobre compasión hacia los animales puede 
diferir en la cantidad de factores involucrados y de ser así, resultaría interesante para futuras investigaciones identificar las variables que podrían estar mediando esta diferencia en el número de factores, además es recomendable que futuros estudios investiguen la percepción de semejanzas y la aceptación o rechazo de cualidades psicológicas entre humanos y animales, debido a que esta parece ser una variable que puede moderar la respuesta del ser humano ante el sufrimiento animal (Bastian, Loughnan, et al., 2012; Bilewicz et al., 2011; Bratanova et al., 2011; Butterfield et al., 2012; Herzog, 2010; Kiesler et al., 2006).

El análisis de correlación de los puntajes de la ECA con las sub-escalas de la ECOM, fue consistente con lo reportado previamente por LópezTello \& Moreno-Coutiño (2019) quienes encontraron una correlación moderada entre reactivos que evaluaban la compasión hacia los animales y hacia los humanos. Esta evidencia de validez de la ECA plantea la importancia de realizar investigaciones que permitan identificar si la compasión hacia los animales se relaciona con conductas especificas del ser humano como la conducta prosocial, preocupación por el otro, agresividad, conductas antisociales, etc., tal como ha sido explorado por diversos autores (Ascione \& Shapiro, 2009; Henderson et al., 2011; McPhedran, 2009; Overton et al., 2012; Preylo \& Arikawa, 2008; Schwartz et al., 2012; Signal \& Taylor, 2007; Taylor \& Signal, 2005).

La ausencia de diferencias significativas en los puntajes de la ECA a partir del género y del nivel educativo parece indicar que estas no son variables que influyan en los niveles de compasión de los participantes, aunque desde luego determinar dichas diferencias no era el objetivo principal del presente estudio, valdría la pena considerar para futuras investigaciones la inclusión de variables como la convivencia con mascotas, el reconocimiento de cualidades psicológicas en los animales o si se desempeña un trabajo que implica contacto frecuente con estos, como variables que podrían explicar mejor los niveles de compasión tal como han sugerido estudios previos (Amiot \& Bastian, 2015; Bastian, Costello, et al., 2012; Epley et al., 2007). En este mismo sentido se vuelve esencial la realización de estudios con mayores tamaños muéstrales y grupos de comparación equilibrados que favorezcan la variabilidad de los datos obtenidos y a su vez permitan la construcción de modelos explicativos sobre el fenómeno.

La evidencia de validez y confiabilidad obtenidas en el presente estudio abren una posibilidad importante sobre el uso de la ECA en diversos ámbitos, por ejemplo, en la investigación clínica en torno a los efectos positivos sobre la salud de personas que mantienen una interacción cercana con un animal de compañía o tienen actitudes positivas hacía ellos (Barker \& Wolen, 2008; Friedmann \& Son, 2009; Headey et al., 2002), encontrando en estos casos menores índices de ansiedad (Odendaal \& Meintjes, 2003; Shiloh et al., 2003; Zilcha-Mano et al., 2012), menor estrés (Allen et al., 2001; Friedmann et al., 2007; Wells, 2005), mayores índices de bienestar (Sugawara et al., 2012) y en general una mejor salud (Headey \& Grabka, 2007). 
Otra área de aplicación de la escala puede darse en la investigación sobre la eficacia de los tratamientos asistidos con animales en donde entre otras variables la compasión hacía los animales y el reconocimiento de sus cualidades psicológicas podría ser una variable moderadora de la eficacia de estas intervenciones (Fine, 2010; McNicholas \& Collis, 2006; Nimer \& Lundah, 2007; O’Haire, 2013; Parish-Plass, 2008; Zilcha-Mano et al., 2011).

La ECA también nos puede proporcionar una medida útil en la investigación sobre los efectos positivos para el bienestar animal derivados de una actitud favorable hacía ellos, lo cual ya ha sido reportado previamente en algunas investigaciones (Kielland et al., 2010; Marinelli et al., 2007; Odendaal \& Lehmann, 2000; Schöberl et al., 2012). Sin embargo, hasta ahora no se ha utilizado una medida sobre compasión, lo cual podría aportar mayor información sobre las variables especificas que favorecen un menor sufrimiento y un mayor bienestar en los animales, esto a su vez permitiría desarrollar estrategias encaminadas a incrementar los niveles de dichas variables en las personas que trabajan con animales (veterinarios, granjeros, trabajadores de zoológicos, etc.) o conviven cotidianamente con uno.

Actualmente existe un cuerpo importante de investigación que respalda las intervenciones centradas en la compasión con el objetivo de incrementar los niveles de esta variable en las personas (Leaviss \& Uttley, 2015). Aunque este tipo de intervenciones hasta ahora no se han utilizado para incrementar la compasión hacía los animales, la existencia de la ECA representa un método de medición que podría contribuir a la evaluación de cambios producidos por una intervención con este objetivo.

Un referente importante de este tipo de intervenciones desde una perspectiva ecológica y en concordancia con lo mencionado por Bekoff (2010, 2013) y Ramp y Bekoff (2015) son los Programas de Educación Humana (HEP por sus siglas en inglés), en los cuáles el objetivo principal es desarrollar actitudes y comportamientos en pro de los animales que a su vez repercutan de manera secundaria en una serie de beneficios para los humanos (Arbour et al., 2009; Nicoll et al., 2008; Thompson \& Gullone, 2003). En este sentido la implementación y eficacia de este tipo de programas en México u otros países podría ser evaluada también por medio de un instrumento con las características de la ECA.

Entre las principales limitaciones del presente estudio la más importante se desprende del origen del instrumento producto de un análisis secundario de un banco de reactivos preexistente, esto puede representar una limitación importante en tanto que la cantidad de reactivos para el análisis exploratorio fue limitado únicamente a los nueve reactivos obtenidos de este análisis secundario reduciendo la posibilidad de estudiar otros factores que pudieran formar parte del constructo, por lo cual es importante continuar explorando la estructura factorial de la compasión hacía los animales por medio del desarrollo de modelos explicativos del fenómeno. Otra limitación del presente estudio es que no se incluyeron grupos poblacionales específicos, que permitieran realizar comparaciones entre personas que podrían estar expuestas con mayor 
frecuencia al sufrimiento animal o con diversas poblaciones clínicas. Esto no permite generalizar los hallazgos aquí reportados, lo cual nos invita a tomarlos con cautela, sin embargo, el presente trabajo abre la posibilidad de realizar más investigación en diferentes poblaciones considerando que la ECA tiene las bondades de ser un instrumento breve, de aplicación sencilla y que podría ser fácilmente adaptado a otros idiomas.

\section{Conclusiones}

La ECA es una herramienta con evidencia de validez y confiabilidad adecuada para evaluar compasión hacia los animales en población mexicana. Su desarrollo representa una aportación novedosa en tanto que es el primer instrumento en español que mide específicamente este constructo.

La presente investigación conforma una primera aproximación a un fenómeno que por sí mismo merece seguir siendo investigado con mayor amplitud y puede constituir un recurso útil en diversos sentidos, por una parte en la evaluación de intervenciones clínicas orientas a incrementar la compasión hacia los animales, ya sea por sus beneficios a la salud humana o en un sentido más amplio, por su impacto social y ambiental en la investigación y evaluación de acciones y políticas dirigidas a la prevención y solución de diversos problemas medio ambientales mediados por la actitud y comportamiento humano hacía los animales.

La apertura de estas posibles líneas de investigación puede representar un sólido punto de apoyo para el desarrollo de programas específicos que busquen que el ser humano desarrolle un sentido más profundo de pertenencia y conexión con la naturaleza y con los animales, con quienes no solo compartimos el espacio físico del mundo, sino la experiencia sensible de dolor, placer, cansancio, satisfacción o insatisfacción que deriva del esfuerzo cotidiano que implica adaptarse y sobrevivir.

\section{Referencias}

Allen, K., Shykoff, B. E., \& Izzo Jr, J. L. (2001). Pet ownership, but not ACE inhibitor therapy, blunts home blood pressure responses to mental stress. Hypertension, 38(4), 815-820. https://doi.org/10.1161/hyp.38.4.815

Allen, M. W., Hunstone, M., Waerstad, J., Foy, E., Hobbins, T., Wikner, B., \& Wirrel, J. (2002). Human-to-animal similarity and participant mood influence punishment recommendations for animal abusers. Society \& Animals: Journal of Human-Animal Studies, 10(3), 267-284. https://do i.org/10.1163/156853002320770074

Amiot, C. E., \& Bastian, B. (2015). Toward a psychology of human-animal relations. Psychological Bulletin, 141(1), 6.47. https://doi.org/10.1037/a 0038147

Amiot, C. E., \& Bastian, B. (2017). Solidarity with animals: Assessing a relevant dimension of social identification with animals. PloS one, 12(1), e0168184. https://doi.org/10.1371/journal.pone.0168184 
Andrukonis, A., Hall, N. J., \& Protopopova, A. (2020). The impact of caring and killing on physiological and psychometric measures of stress in animal shelter employees: A pilot study. International Journal of Environmental Research and Public Health, 17(24), 9196. https://doi.org/10.3390/ijerp h17249196

Arbour, R., Signal, T., \& Taylor, N. (2009). Teaching kindness: The promise of humane education. Society \& Animals, 17(2), 136-138. https://doi.org/1 $0.1163 / 156853009 X 418073$

Ascione, F., \& Shapiro, K. (2009). People and animals, kindness and cruelty: Research directions and policy implications. Journal of Social Issues, 65(3), 569-587.

Barker, S. B., \& Wolen, A. R. (2008). The benefits of human-companion animal interaction: A review. Journal of Veterinary Medical Education, 35(4), 487-495. https://doi.org/10.3138/jvme.35.4.487

Bastian, B., Costello, K., Loughnan, S., \& Hodson, G. (2012). When closing the human-animal divide expands moral concern: The importance of framing. Social Psychological and Personality Science, 3(4), 421-429. https ://doi.org/10.1177/1948550611425106

Bastian, B., Loughnan, S., Haslam, N., \& Radke, H. R. (2012). Don't mind meat? The denial of mind to animals used for human consumption. Personality and Social Psychology Bulletin, 38(2), 247-256. https://doi.or $\mathrm{g} / 10.1177 / 0146167211424291$

Bekoff, M. (2010). Conservation lacks compassion. New Scientist, 207(2775), 24-25. https://doi.org/10.1016/S0262-4079(10)62080-5

Bekoff, M. (2013). Ignoring nature no more: The case for compassionate conservation. University of Chicago Press. https://doi.org/10.7208/9780 226925363

Bilewicz, M., Imhoff, R., \& Drogosz, M. (2011). The humanity of what we eat: Conceptions of human uniqueness among vegetarians and omnivores. European Journal of Social Psychology, 41(2), 201-209. https://doi.org/10 $.1002 /$ ejsp.766

Bonermann, B., \& Singer, T. (2013). A cognitive neuroscience perspective. The resource model of compassion. En T. Singer \& M. Bolz (Eds.), Compassion. Bridging practice and science (pp. 179-191). Max Planck Institute for Human Cognitive and Brain Sciences.

Bratanova, B., Loughnan, S., \& Bastian, B. (2011). The effect of categorization as food on the perceived moral standing of animals. Appetite, 57(1), 193-196. https://doi.org/10.1016/j.appet.2011.04.020

Butterfield, M. E., Hill, S. E., \& Lord, C. G. (2012). Mangy mutt or furry friend? Anthropomorphism promotes animal welfare. Journal of Experimental Social Psychology, 48(4), 957-960. https://doi.org/10.1016/j.jesp.2012.0 2.010

Byrne, B. M. (2001) Structural equation modeling with AMOS: Basic concepts, applications, and programming. Routledge.

Caviola, L., Everett, J. A. C., \& Faber, N. S. (2019). The moral standing of animals: Towards a psychology of speciesism. Journal of Personality and Social Psychology, 116(6), 1011-1029. https://doi.org/10.1037/pspp000 0182 
Chang, J. H., Fresco, J., \& Green, B. (2014). The development and validation of the Compassion of Others' Lives Scale (the COOL Scale). International Journal of Humanities and Social Science, 4(5), 33-42.

Costello, K., \& Hodson, G. (2010). Exploring the roots of dehumanization: The role of animal-human similarity in promoting immigrant humanization. Group Processes \& Intergroup Relations, 13(1), 3-22. https://doi.org/10.1 $177 / 1368430209347725$

Davidson, R., \& Harrington, A. (2002). Visions of compassion: Western scientists and Tibetan Buddhists examine human nature. Oxford University Press.

Dhont, K., Hodson, G., Costello, K., \& MacInnis, C. C. (2014). Social dominance orientation connects prejudicial human-human and humananimal relations. Personality and Individual Differences, 61-62, 105-108. https://doi.org/10.1016/j.paid.2013.12.020

Epley, N., Waytz, A., \& Cacioppo, J. T. (2007). On seeing human: A threefactor theory of anthropomorphism. Psychological Review, 114(4), 864886. https://doi.org/10.1037/0033-295X.114.4.864

Fine, A. H. (2010). Incorporating animal-assisted therapy into psychotherapy: Guidelines and suggestions for therapists. En A. H. Fine (Ed.), Animalassisted therapy: Theoretical foundations and guidelines for practice (3. ed., pp. 169-191). Academic Press. https://doi.org/10.1016/B978-0-12-3814 53-1.10010-8

Friedmann, E., \& Son, H. (2009). The human-companion animal bond: how humans benefit. Veterinary Clinics of North America: Small Animal Practice, 39(2), 293-326. https://doi.org/10.1016/j.cvsm.2008.10.015

Friedmann, E., Thomas, S. A., Cook, L. K., Tsai, C. C., \& Picot, S. J. (2007). A friendly dog as potential moderator of cardiovascular response to speech in older hypertensives. Anthrozoös, 20(1), 51-63. https://doi.org/10.275 2/089279307780216605

Gamboa-Bernal, G. A. (2011). Ecología humana y ecología ambiental: binomio clave. PErSona y bioÉtica, 15(1), 5-9.

Germer, C. K., \& Siegel, R. D. (2012). Wisdom and compassion in psychotherapy: Deepening mindfulness in clinical practice. The Guilford Press.

Gilbert, P. (2013). The flow of life. An evolutionary model of compassion. En T. Singer \& M. Bolz (Eds.), Compassion. Bridging practice and science (pp. 127-149). Max Planck Institute for Human Cognitive and Brain Sciences.

Gilbert, P., \& Choden. (2013). Mindful compassion. Using the power of mindfulness and compassion to transform our lives. Hachette.

Goetz, J. L., Keltner, D., \& Simon-Thomas, E. (2010). Compassion: An evolutionary analysis and empirical review. Psychological Bulletin, 136(3), 351-374. https://doi.org/10.1037/a0018807

Hawkins, R. D., \& Williams, J. M. (2016). Children's beliefs about animal minds (Child-BAM): Associations with positive and negative childanimal interactions. Anthrozoös, 29(3), 503-519. https://doi.org/10.108 0/08927936.2016.1189749

Hawkins, R. D., \& Williams, J. M. (2017). Childhood attachment to pets: Associations between pet attachment, attitudes to animals, compassion, and humane behaviour. International Journal of Environmental Research and Public Health, 14(5), 490. https://doi.org/10.3390/ijerph 14050490 
Headey, B., \& Grabka, M. M. (2007). Pets and human health in Germany and Australia: National longitudinal results. Social Indicators Research, 80(2), 297-311. https://doi.org/10.1007/s11205-005-5072-z

Headey, B., Grabka, M., Kelley, J., Reddy, P., \& Tseng, Y. P. (2002). Pet ownership is good for your health and saves public expenditure too: Australian and German longitudinal evidence. Australian Social Monitor, 5(4), 93-99.

Henderson, B. B., Hensley, C., \& Tallichet, S. E. (2011). Childhood animal cruelty methods and their link to adult interpersonal violence. Journal of Interpersonal Violence, 26(11), 2211-2227. https://doi.org/10.1177/088 6260510383038

Herzog, H. (2010). Some we love, some we hate, some we eat: Why it's so hard to think straight about animals. Harper.

Herzog, H. (2011). The impact of pets on human health and psychological well-being: Fact, fiction, or hypothesis? Current Directions in Psychological Science, 20(4), 236-239. https://doi.org/10.1177/0963721411415220

Kielland, C., Skjerve, E., Osterås, O., \& Zanella, A. J. (2010). Dairy farmer attitudes and empathy toward animals are associated with animal welfare indicators. Journal of Dairy Science, 93(7), 2998-3006. https://doi.org/1 $0.3168 /$ jds.2009-2899

Kiesler, S., Lee, S. L., \& Kramer, A. D. (2006). Relationship effects in psychological explanations of nonhuman behavior. Anthrozoös, 19(4), 335-352.

Kline, R. (2005). Principles and practices of structural equation modeling. Guilford Press.

Leaviss, J., \& Uttley, L. (2015). Psychotherapeutic benefits of compassionfocused therapy: An early systematic review. Psychological Medicine, 45(5), 927-945. http://doi.org/10.1017/S0033291714002141.

López-Tello, A., \& Moreno-Coutiño, A. B. (2019). Escala de Compasión (ECOM) para población mexicana. Psicología y Salud, 29(1), 25-32. http s://doi.org/10.25009/pys.v29i1.2565

Marinelli, L., Adamelli, S., Normando, S., \& Bono, G. (2007). Quality of life of the pet dog: Influence of owner and dog's characteristics. Applied Animal Behaviour Science, 108(1-2), 143-156. https://doi.org/10.1016/j.applani m.2006.11.018

Martins, D., Nicholas, N.A., Shaheen, M., Jones, L., \& Norris, K. (2013). The development and evaluation of a compassion scale. Journal of Health Care for the Poor and Underserved, 24(3), 1235-1246. https://doi.org/10.1353 /hpu.2013.0148

McNicholas, J., \& Collis, G. M. (2006). Animals as social supports: Insights for understanding animal-assisted therapy. En A. H. Fine (Ed.), Handbook on animal-assisted therapy: Theoretical foundations and guidelines for practice ( $2^{a}$ ed., pp. 49-71). Academic Press.

McPhedran, S. (2009). Animal abuse, family violence, and child wellbeing: A review. Journal of Family Violence, 24(1), 41-52. https://doi.org/10.1007 /s10896-008-9206-3

Mercadillo, R. (2012). Retratos del cerebro compasivo: una reflexión en la neurociencia social, los policias y el género. Centro de Estudios Filosóficos, Políticos y Sociales Vicente Lombardo Toledano. 
Neff, K. D. (2003). The development and validation of a scale to measure selfcompassion. Self and Identity, 2(3), 223-250. https://doi.org/10.1080/1 5298860309027

Nicoll, K., Samuels, W. E., \& Trifone, C. (2008). An in-class, humane education program can improve young students' attitudes toward animals. Society \& Animals, 16(1), 45-60. https://doi.org/10.1163/156853008X269881

Nimer, J., \& Lundahl, B. (2007). Animal-assisted therapy: A meta-analysis. Anthrozoös, 20(3), 225-238. https://doi.org/10.2752/089279307X2247 73

O'Haire, M. E. (2013). Animal-assisted intervention for autism spectrum disorder: A systematic literature review. Journal of Autism and Developmental Disorders, 43(7), 1606-1622. https://doi.org/10.1007/s1 0803-012-1707-5

Odendaal, J. S., \& Lehmann, S. M. C. (2000). The role of phenylethylamine during positive human-dog interaction. Acta Veterinaria Brno, 69(3), 183-188. http://dx.doi.org/10.2754/avb200069030183

Odendaal, J. S., \& Meintjes, R. A. (2003). Neurophysiological correlates of affiliative behaviour between humans and dogs. The Veterinary Journal, 165(3), 296-301. https://doi.org/10.1016/S1090-0233(02)00237-X

Overton, J. C., Hensley, C., \& Tallichet, S. E. (2012). Examining the relationship between childhood animal cruelty motives and recurrent adult violent crimes toward humans. Journal of Interpersonal Violence, 27(5), 899-915. https://doi.org/10.1177/0886260511423256

Ozawa-de Silva, B. R., Dodson-Lavelle, B., Raison, C. L., Negi, L. T., Silva, B. R. O., \& Phil, D. (2012). Compassion and ethics: Scientific and practical approaches to the cultivation of compassion as a foundation for ethical subjectivity and well-being. Journal of Healthcare, Science and the Humanities, 2(1), 145-161.

Parish-Plass, N. (2008). Animal-assisted therapy with children suffering from insecure attachment due to abuse and neglect: A method to lower the risk of intergenerational transmission of abuse? Clinical Child Psychology and Psychiatry, 13(1), 7-30. https://doi.org/10.1177/1359104507086338

Phillips, A., \& Taylor, B. (2009). On kindness. Hamish Hamilton Press.

Preylo, B. D., \& Arikawa, H. (2008). Comparison of vegetarians and nonvegetarians on pet attitude and empathy. Anthrozoös, 21(4), 387-395. htt ps://doi.org/10.2752/175303708X371654

Ramp, D., \& Bekoff, M. (2015). Compassion as a practical and evolved ethic for conservation. BioScience, 65(3), 323-327. https://doi.org/10.1093/biosci /biu223

Ricard, M. (2017). En defensa de los animales. Editorial Kairós.

Schöberl, I., Wedl, M., Bauer, B., Day, J., Möstl, E., \& Kotrschal, K. (2012). Effects of owner-dog relationship and owner personality on cortisol modulation in human-dog dyads. Anthrozoös, 25(2), 199-214. https://d oi.org/10.2752/175303712X13316289505422

Schwartz, R. L., Fremouw, W., Schenk, A., \& Ragatz, L. L. (2012). Psychological profile of male and female animal abusers. Journal of Interpersonal Violence, 27(5), 846-861. https://doi.org/10.1177/0886260511423254 
Shiloh, S., Sorek, G., \& Terkel, J. (2003). Reduction of state-anxiety by petting animals in a controlled laboratory experiment. Anxiety, Stress, and Coping, 16(4), 387-395. https://doi.org/10.1080/1061580031000091582

Signal, T. D., \& Taylor, N. (2007). Attitude to animals and empathy: Comparing animal protection and general community samples. Anthrozoös, 20(2), 125-130. http://dx.doi.org/10.2752/175303707X20 7918

Singer, T., \& Klimecki, O. M. (2014). Empathy and compassion. Current Biology, 24(18), R875-R878. https://doi.org/10.1016/j.cub.2014.06.054

Sprecher, S., \& Fehr, B. (2005). Compassionate love for close others and humanity. Journal of Social and Personal Relationships, 22(5), 629-651. h ttps://doi.org/10.1177/0265407505056439

Sugawara, A., Masud, M. M., Yokoyama, A., Mizutani, W., Watanuki, S., Yanai, K., ... \& Tashiro, M. (2012). Effects of presence of a familiar pet dog on regional cerebral activity in healthy volunteers: A positron emission tomography study. Anthrozoös, 25(1), 25-34. http://dx.doi.org/10.2752/ $175303712 \times 13240472427311$

Taylor, N., \& Signal, T. D. (2005). Empathy and attitudes to animals. Anthrozoös, 18(1), 18-27. http://dx.doi.org/10.2752/089279305785594 342

Thompson, K., \& Gullone, E. (2003). The children's treatment of animals questionnaire (CTAQ): A psychometric investigation. Society \& Animals, 11(1), 1-15. https://doi.org/10.1163/156853003321618800

Valera, L. (2019). Ecología humana. Nuevos desafíos para la ecología y la filosofía. Arbor, 195(792), a509. https://doi.org/10.3989/arbor.2019.79 $2 \mathrm{n} 2010$

Vega, C. (2015). Papel de trabajo, Aspectos epistemológicos de la estimación estadística de modelos: investigación ex-post-facto. Procesos Estocaticos Del IMYCA, 1-8. http://doi.org/10.13140/RG.2.1.3098.0647

Wells, D. L. (2005). The effect of videotapes of animals on cardiovascular responses to stress. Stress and Health, 21(3), 209-213. http://doi.org/10. $1002 /$ smi.1057

Zilcha-Mano, S., Mikulincer, M., \& Shaver, P. R. (2011). Pet in the therapy room: An attachment perspective on animal-assisted therapy. Attachment \& Human Development, 13(6), 541-561. http://doi.org/10.1080/14616 734.2011 .608987

Zilcha-Mano, S., Mikulincer, M., \& Shaver, P. R. (2012). Pets as safe havens and secure bases: The moderating role of pet attachment orientations. Journal of Research in Personality, 46(5), 571-580. http://doi.org/10.1016/j.jrp.2 012.06 .005 